04

\title{
Оптимизация потерь мощности атомарного пучка при его транспортировке в плазму токамака ТУМАН-ЗМ
}

\author{
() В.А. Корнев, Г.И. Абдуллина, Л.Г. Аскинази, А.А. Белокуров, Н.А. Жубр, С.В. Лебедев, \\ Д.В. Разуменко, А.С. Тукачинский
}

Физико-технический институт им. А.Ф. Иофффе РАН, Санкт-Петербург, Россия

E-mail: Vladimir.Kornev@mail.ioffe.ru

Поступило в Редакцию 24 ноября 2020 г.

В окончательной редакции 24 ноября 2020 г.

Принято к публикации 25 ноября 2020 г.

\begin{abstract}
Представлены результаты анализа механизмов потерь энергичного атомарного пучка в соединительном тракте токамака ТУМАН-3М. Построена численная модель, описывающая эффект снижения коэффициента пропускания и позволяющая определить пути снижения потерь. С учетом результатов расчета разработан тракт с увеличенным поперечным сечением. Обнаружено, что замена тракта привела к 1.5-2-кратному росту интегрального нейтронного потока, обусловленного dd-реакциями энергичных дейтронов с мишенной плазмой.
\end{abstract}

Ключевые слова: высокотемпературная плазма, токамак, инжекция атомарного пучка, высокоэнергичные частицы, генерация термоядерных нейтронов.

DOI: 10.21883/PJTF.2021.06.50748.18629

Широко обсуждаемая в последнее время концепция источника термоядерных нейтронов на базе компактного токамака [1-3] основана на генерации нейтронов при инжекции пучка высокоэнергичных частиц. Ввиду ограниченности размеров такого токамака актуальность проблемы транспортировки атомарного пучка большой мощности существенно возросла. Проблема потерь мощности пучка высокоэнергичных атомов при транспортировке от источника к разрядной камере токамака была идентифицирована в первых экспериментах по инжекционному нагреву плазмы [4,5]. В настоящей работе представлены результаты исследования возможности снижения потерь атомарного пучка в соединительном тракте токамака ТУМАН-3М с целью достижения оптимального пропускания.

Эксперименты выполнялись на токамаке ТУМАН-3М [6] с использованием комплекса инжекционного нагрева, позволяющего вводить в плазму пучки нейтральных атомов водорода/дейтерия, ускоренных до энергии $24 \mathrm{keV}$ [7]. В первых экспериментах инжекционный комплекс был оборудован дуговым ионным источником ИПМ-2 (источник с периферийным магнитным полем), рассчитанным на ионные токи до $25 \mathrm{~A}$. Атомарный пучок от инжектора к камере токамака доставлялся через соединительный тракт с эллиптическим поперечным сечением $67 \times 205 \mathrm{~mm}$. В этих условиях при инжекции были зарегистрированы двукратный нагрев ионов и рост интегрального потока $2.45 \mathrm{MeV}$ $\mathrm{dd}$-нейтронов $R_{n}$ до $10^{11} \mathrm{~s}^{-1}[8]$. В этих экспериментах при максимальной энергии инжекции наблюдалось насыщение роста нейтронного выхода [9], причиной которого могло быть уменьшение пропускания тракта. С целью повышения нейтронного выхода в 2015 г. инжектор был дооборудован более мощным ионным источником ИПМ-1, позволяющим увеличить ионный ток в 1.5-2 раза. В экспериментах с источником ИПМ-1 при увеличении мощности инжекции было обнаружено насыщение прироста ионной температуры $T_{i}(0)$ и нейтронного выхода $R_{n}$ [9]. Также наблюдалось уменьшение потоков энергичных нейтральных атомов перезарядки, что указывало на снижение количества быстрых ионов [10]. Для объяснения наблюдаемых явлений были предложены две гипотезы: 1) рост орбитальных потерь, приводящий к увеличению количества примесей, поступающих со стенок и разбавляющих фоновую плазму; 2) „запирание“ нейтрального пучка в соединительном тракте из-за роста плотности остаточного газа в соответствии с механизмом $[4,5]$. В рамках данного исследования проверялась вторая из упомянутых гипотез.

Оценки потерь пучка в тракте проводились с помощью расчетов, учитывающих ослабление пучка при взаимодействии с остаточным газом [4]. При этом эквивалентный ток быстрых атомов пучка $I_{p a s s}$, прошедших через тракт, принимался равным

$$
I_{\text {pass }}=I_{0}(1-\alpha) \exp (-n \sigma L) .
$$

Здесь $I$ - число нейтральных атомов, проходящих через поперечное сечение тракта в единицу времени, $I$ имеет размерность A/C (ампер/кулон), $I_{\text {pass }}$ - ток пучка после тракта, $I_{0}$ - ток пучка перед трактом, $\alpha$ - доля быстрых атомов, попадающих на стенки из-за „обрезания“ размера пучка входным патрубком тракта, $n$ - концентрация остаточного газа в тракте, $\sigma$ - сечение ионизации быстрых атомов пучка при столкновениях с молекулами остаточного газа, $L-$ длина тракта. В рамках данной модели изменение кон- 


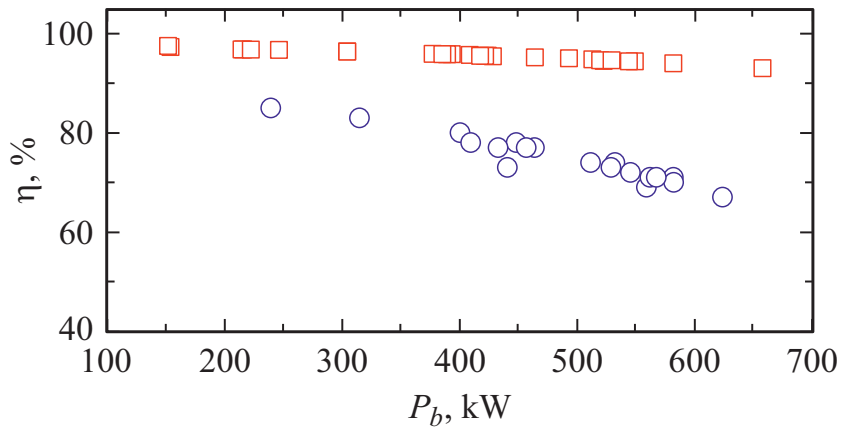

Рис. 1. Зависимость коэффициента пропускания $\eta$ от мощности на входе трактов с меньшим (эллиптическим) сечением (кружки) и с увеличенным (прямоугольным) сечением (квадраты).

центрации остаточного газа $n$ в соединительном тракте описывается следующим уравнением:

$$
\begin{aligned}
V d n / d t & =\alpha I_{0} \gamma+(1-\alpha) I_{0} \gamma[1-\exp (-n \sigma L)] \\
& -c_{e f f}\left(n-n_{0}\right)
\end{aligned}
$$

Здесь $V$ - объем тракта, $\gamma$ - число молекул газа, выбиваемых из стенки тракта быстрым ионом или атомом (в расчетах в соответствии с [11] была принята оценка $\gamma=2.5$ для диапазона энергий $12-24 \mathrm{keV}$, характерного для описываемых экспериментов), $n_{0}$ - концентрация газа в тракте перед включением инжекции, $c_{e f f}-$ эффективная скорость откачки газа из тракта. Эффективная скорость откачки принималась равной $4.5 c_{c o n}$, что составляет $2.8 \mathrm{~m}^{3} / \mathrm{s}$ для эллиптического патрубка. Здесь $c_{c o n}$ - проводимость тракта, вычисленная согласно [12]. Расчет траекторий ионов, ускоряемых в ионно-оптической системе инжектора, позволил определить долю $\alpha$ быстрых атомов, попадающих на стенки тракта. Для эллиптического патрубка величина коэффициента $\alpha$ оказалась равной 0.05 .

Анализ уравнения (2) показал, что на начальной стадии прохождения атомарного пучка через тракт второе и третье слагаемые в правой части малы. Доминирующим процессом, приводящим к снижению пропускания и в то же время к росту концентрации остаточного газа, оказывается „обрезание“ пучка на входе в соединительный тракт - первое слагаемое в правой части уравнения (2). С целью снижения взаимодействия нейтрального пучка со стенками тракта был разработан, изготовлен и установлен на токамак соединительный тракт с увеличенным поперечным сечением (прямоугольное сечение $120 \times 300 \mathrm{~mm})$. В результате увеличения поперечного сечения возросла эффективная скорость откачки газа из тракта $c_{e f f}$ до величины $4.2 \mathrm{~m}^{3} / \mathrm{s}$, а также объем соединительного тракта $V=0.022 \mathrm{~m}$. Расчеты траекторий ионов показали, что в тракте с увеличенным сечением доля быстрых атомов, попадающих на стенки, снизилась до 0.01 .
С помощью уравнений (1) и (2) для ионного источника ИПМ-1 рассчитывалась эволюция коэффициента пропускания нейтрального пучка $\eta(t)=I_{\text {pass }} / I_{0}$ для двух конфигураций тракта при токах и ускоряющих напряжениях, используемых в экспериментах. Для сравнения потерь в двух трактах бралась величина $\eta$ на $10 \mathrm{~ms}$ после начала инжекции, когда, согласно расчетам, достигалось равновесное пропускание. Отметим, что измеренный нейтронный выход, как правило, к этому моменту времени достигал своего максимального значения. На рис. 1 представлена зависимость $\eta$ на $10 \mathrm{~ms}$ импульса инжекции от мощности дейтериевого пучка на входе в тракт. Как видно, увеличение мощности пучка до $600 \mathrm{~kW}$ в тракте с менышим поперечным размером приводит к снижению коэффициента пропускания до 65\%. В то же время в тракте большего сечения в широком диапазоне мощности $\eta$ снижается незначительно и при мощности $650 \mathrm{~kW}$ оказывается не менее $92 \%$.

Увеличение коэффициента пропускания тракта при использовании патрубка с увеличенным сечением было подтверждено измерениями интегрального потока нейтронов при инжекции дейтериевого пучка в дейтериевую плазму. Измерения потока $2.45 \mathrm{MeV}$ нейтронов $R_{n}$ осуществлялись с помощью детекторов, состоящих из ${ }^{3}$ Не-газоразрядных счетчиков и полиэтиленовых замедлителей [13], в разрядах с плазменным током $177 \mathrm{kA}$, средней электронной плотностью $2 \cdot 10^{19} \mathrm{~m}^{-3}$ при индукции продольного магнитного поля 1.0 Т. Результаты этих измерений представлены на рис. 2. Из приведенных рисунков видно, что во всем исследованном диапазоне мощностей нейтронный выход при использовании патрубка с увеличенным сечением оказывается в 1.5-2.0 раза больше. Поскольку в этой серии экспериментов плотность фоновой плазмы оставалась неизменной, весь прирост $R_{n}$ следует связать с увеличившимся количе-

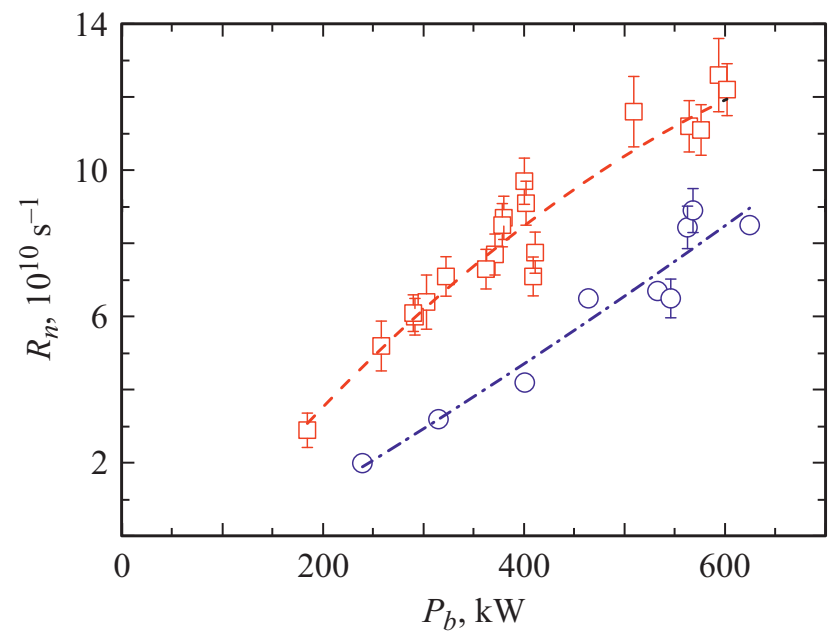

Рис. 2. Зависимость интегрального потока нейтронов $R_{n}$ от мощности пучка $P_{b}$ на выходе инжектора при использовании соединительного патрубка меньшего (эллиптического) сечения (кружки) и патрубка большего (прямоугольного) сечения (квадраты). 

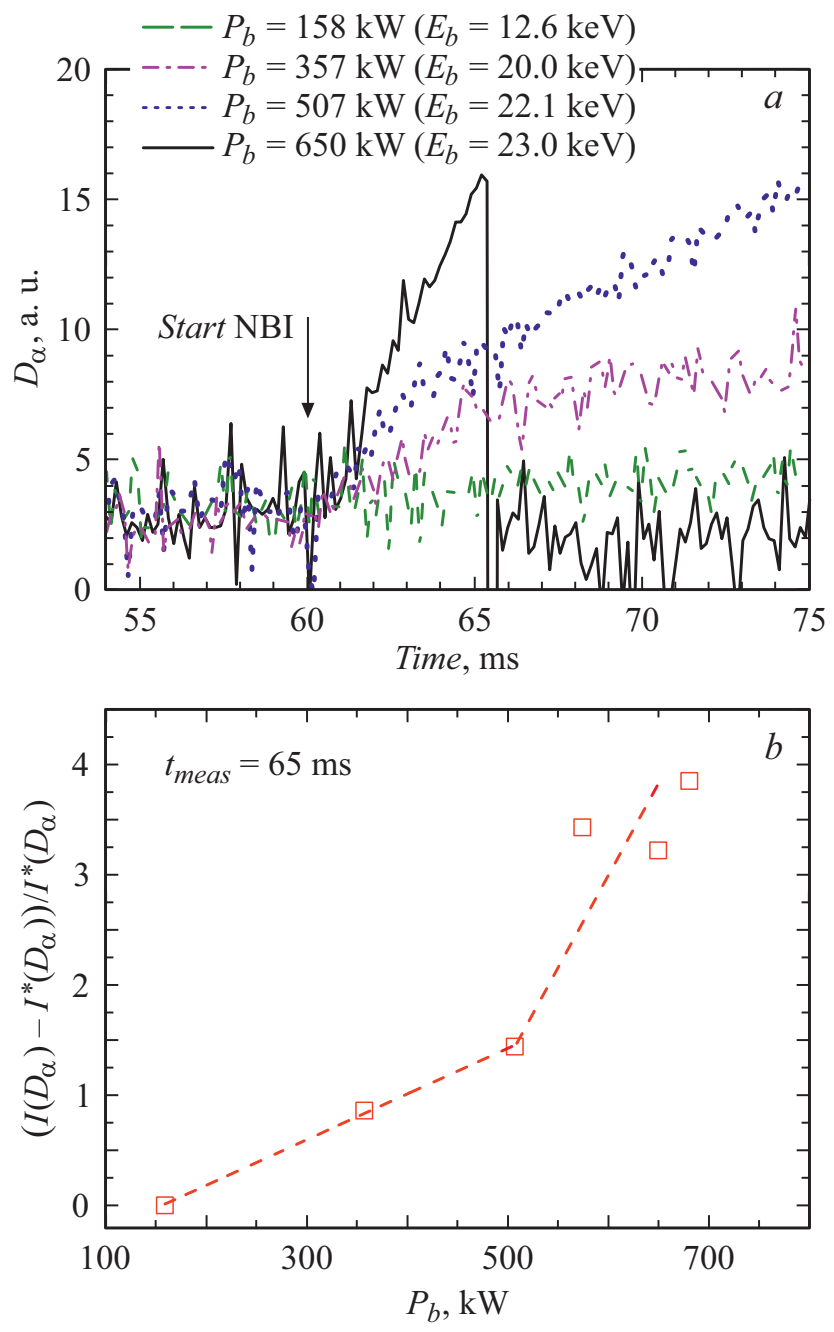

Рис. 3. $a-$ эволюция интенсивности $D_{\alpha}$ в соединительном патрубке большего (прямоугольного) сечения при инжекции атомарного пучка различной мощности (NBI - neutral beam injection); $b$ - относительный рост интенсивности свечения линии $D_{\alpha}$ в зависимости от мощности пучка $P_{b}$.

ством энергичных дейтронов, что находится в согласии с увеличением коэффициента пропускания прямоугольного патрубка. Максимальный нейтронный выход при этом достиг $1.2 \cdot 10^{11} \mathrm{~s}^{-1}$.

Новый тракт оборудован двумя диагностическими патрубками, позволяющими осуществить спектроскопические измерения и, в частности, наблюдение интенсивности излучения линии $D_{\alpha}$, пропорциональной потоку нейтрального газа со стенок тракта. Результаты измерений интенсивности $D_{\alpha}$ при использовании источника ИПМ-1 приведены на рис. 3, $a$. При инжекции атомарного пучка с мощностью менее $160 \mathrm{~kW}$ в присутствии плазмы, а также магнитных полей плазменного тока и токов в обмотках равновесия влияния инжекции на интенсивность свечения в тракте не наблюдалось (штриховая линия). Отметим, что ненулевой уровень $D_{\alpha}$, существующий еще до начала инжекции, обусловлен свечением, возникающим в плазме и попадающим в систему детектирования в результате переотражений от стенок тракта. При дальнейшем увеличении мощности инжекции наблюдался рост интенсивности $D_{\alpha}$ (штрихпунктирная, пунктирная и сплошная линии на рис. $3, a)$. Рост интенсивности $D_{\alpha}$ указывает на наличие взаимодействия частиц пучка со стенками тракта, приводящего к заметной десорбции газа со стенок, и соответствующего роста концентрации остаточного газа. Отметим, что в представленных случаях наблюдается линейный рост интенсивности, что свидетельствует о справедливости сделанной выше оценки, указывающей на доминирующую роль первого слагаемого в правой части уравнения (2) на начальной стадии инжекции.

На рис. $3, b$ представлена зависимость относительного прироста интенсивности свечения $\left(I\left(D_{\alpha}\right)-I^{*}\left(D_{\alpha}\right)\right) / I^{*}\left(D_{\alpha}\right)$ от мощности на входе в соединительный тракт $P_{b}$. Здесь $I^{*}\left(D_{\alpha}\right)$ и $I\left(D_{\alpha}\right)$ - интенсивность $D_{\alpha}$ перед началом инжекции и на $5 \mathrm{~ms}$ инжекции (65 ms разряда) соответственно. Видно, что при росте мощности до $500 \mathrm{~kW}$ прирост остается линейным, тогда как при дальнейшем увеличении мощности прирост $I\left(D_{\alpha}\right)$ резко возрастает, что, очевидно, свидетельствует о резком возрастании концентрации остаточного газа $n$ за счет интенсивного взаимодействия со стенками атомов и ионов, отклоняющихся в тракте, - процесса, учитываемого вторым слагаемым в правой части уравнения (2). Резкое увеличение концентрации остаточного газа при мощности выше $500 \mathrm{~kW}$ приводит к уменьшению коэффициента пропускания атомарного пучка соединительным трактом в 1.5-2 раза. Факт уменьшения пропускания атомарного пучка подтверждается экспериментальным наблюдением замедления роста нейтронного выхода при $P_{b}>500 \mathrm{~kW}$ (рис. 2). Здесь видно, что наклон верхней кривой, показывающей зависимость интегрального потока нейтронов от мощности нейтрального пучка на входе в соединительный тракт большего (прямоугольного) сечения, уменьшается при больших мощностях $P_{b}$.

В заключение следует отметить еще одно обстоятельство, которое может иметь значение для снижения потерь в транспортировочном тракте токамака. Результаты наблюдения эволюции интенсивности свечения $D_{\alpha}$ в токамаке ТУМАН-3М (рис. 3) указывают на интенсивную ионизацию дейтерия в соединительном тракте: действительно, сечения ионизации и возбуждения пропорциональны в широком диапазоне скоростей взаимодействующих частиц, а значит, наряду с возбуждением происходит и ионизация атомов. Для образующихся в результате ионизации быстрых ионов помимо столкновительного механизма отклонения от первоначальной траектории может оказаться существенным отклонение магнитным полем плазменного тока и обмоток управления. Доля частиц, попадающих на стенку за счет этого эффекта, в представленном исследовании не была установлена и будет предметом дальнейшего изучения.

Таким образом, обнаружено, что при ограниченном поперечном сечении соединительного тракта наблюдает- 
ся заметное снижение пропускания энергичного атомарного пучка, приводящее, в частности, к уменьшению нейтронного выхода из плазмы компактного токамака. Замена соединительного тракта на тракт с увеличенным сечением привела к 1.5-2-кратному росту нейтронного потока. При максимальных мощностях инжекции наблюдалось замедление роста нейтронного потока с мощностью. Построена численная модель, описывающая наблюдаемый эффект снижения коэффициента пропускания тракта и позволяющая определить пути снижения потерь в соединительном тракте токамака.

\section{Благодарности}

Авторы благодарят ФТИ им. А.Ф. Иоффе за постоянное содействие в поддержке инфраструктуры токамака ТУМАН-3М.

\section{Финансирование работы}

Исследование выполнено при поддержке Российского научного фонда (грант № 16-12-10285-П).

\section{Конфликт интересов}

Авторы заявляют, что у них нет конфликта интересов.

\section{Список литературы}

[1] A.M. Garofalo, M.A. Adboud, J.M. Cankie, V.S. Chan, A.W. Hyatt, D.N. Hill, N.B. Morley, G.A. Navratil, M.E. Sawan, T.S. Taylor, C.P.C. Wong, W. Wu, A. Ying, Fusion Eng. Design, 89, 876 (2014). http://dx.doi.org/10.1016/j.fusengdes.2014.03.055

[2] J.E. Menard, T. Brown, L. El-Guebaly, M. Boyer, J. Canik, B. Colling, R. Raman, Z. Wang, Y. Zhai, P. Buxton, B. Covele, C. D'Angelo, A. Davis, S. Gerhardt, M. Gryaznevich, M. Harb, T.C. Hender, S. Kaye, D. Kingham, M. Kotschenreuther, S. Mahajan, R. Maingi, E. Marriott, E.T. Meier, L. Mynsberge, C. Neumeyer, M. Ono, J.-K. Park, S.A. Sabbagh, V. Soukhanovskii, P. Valanju, R. Woolley, Nucl. Fusion, 56, 106023 (2016). DOI: 10.1088/0029-5515/56/10/106023

[3] B.V. Kuteev, Yu.S. Shpanskiy and DEMO-FNS Team, Nucl. Fusion, 57, 076039 (2017). https://doi.org/10.1088/1741-4326/aa6dcb

[4] A.C. Riviere, J. Sheffield, Nucl. Fusion, 15, 944 (1975).

[5] V.M. Kulygin, A.A. Panasenkov, in Proc. of the 8th Symp. on engineering problems of fusion research (San Francisco, 1979), vol. II, p. 850.

[6] S.V. Lebedev, M.V. Andrejko, L.G. Askinazi, V.E. Golant, V.A. Kornev, S.V. Krikunov, L.S. Levin, B.M. Lipin, G.T. Razdobarin, V.A. Rozhansky, Plasma Phys. Control Fusion, 38, 1103 (1996).
[7] L.G. Askinazi, A.G. Barsukov, V.E. Golant, V.K. Gusev, V.V. Kusnetsov, S.V. Lebedev, V.M. Leonov, A.V. Lupin, V.B. Minaev, A.A. Panasenkov, A.R. Polevoi, G.N. Tilinin, A.S. Tukachinsky, V.A. Yagnov, Plasma Devices Oper., 11, 211 (2003). DOI: 10.1080/1051999031000155524

[8] L.G. Askinazi, F.V. Chernyshev, V.E. Golant, M.A. Irzak, V.A. Kornev, S.V. Krikunov, S.V. Lebedev, A.D. Melnik, D.V. Razumenko, V.V. Rozhdestvensky, A.A. Rushkevich, A.I. Smirnov, A.S. Tukachinsky, M.I. Vildjunas, N.A. Zhubr, in Proc. 34th EPS Conf. on plasma physics (Warsaw, 2007), vol. $31 \mathrm{~F}$, p. 1.146.

[9] V.A. Kornev, L.G. Askinazi, A.A. Belokurov, F.V. Chernyshev, S.V. Lebedev, A.D. Melnik, A.A. Shabelsky, A.S. Tukachinsky, N.A. Zhubr, Nucl. Fusion, 57, 126005 (2017). https://doi.org/10.1088/1741-4326/aa7d13

[10] А.Д. Мельник, Л.Г. Аскинази, А.А. Белокуров, П.Р. Гончаров, В.А. Корнев, С.В. Крикунов, С.В. Лебедев, М.И. Миронов, А.С. Тукачинский, Ф.В. Чернышев, в сб. XLIII Междунар. Звенигород. конф. по физике плазмы и УТС (Звенигород, 2016), с. 89 (М-28).

[11] Н.Н. Семашко, А.Н. Владимиров, В.В. Кузнецов, В.М. Кулыгин, А.А. Панасенков, Инжсекторы быстрых атомов водорода (Энергоиздат, М., 1981).

[12] А.В. Юрьева, Проектирование вакуумных систем (Изд-во ТПУ, Томск, 2012).

[13] В.А. Корнев, Л.Г. Аскинази, М.И. Вильджюнас, Н.А. Жубр, С.В. Крикунов, С.В. Лебедев, Д.В. Разуменко, В.В. Рождественский, А.С. Тукачинский, Письма в ЖТФ, 39 (6), 41 (2013). [Пер. версия: https://doi.org/10.1134/S106378501303022X]. 